\title{
A Prototype Representation to Approximate White Matter Bundles with Weighted Currents
}

\author{
Pietro Gori $^{1}$, Olivier Colliot ${ }^{1}$, Linda Marrakchi-Kacem ${ }^{1,2}$, Yulia Worbe $^{1}$, \\ Fabrizio De Vico Fallani ${ }^{1}$, Mario Chavez ${ }^{1}$, Sophie Lecomte ${ }^{1,2}$, Cyril Poupon ${ }^{2}$, \\ Andreas Hartmann ${ }^{1}$, Nicholas Ayache ${ }^{3}$, and Stanley Durrleman ${ }^{1}$ \\ 1 Inria Paris-Rocquencourt, Sorbonne Universités, UPMC Univ Paris 06 UMR \\ S1127, Inserm U1127, CNRS UMR 7225, ICM, F-75013, Paris, France \\ ${ }^{2}$ Neurospin, CEA, Gif-Sur-Yvette, France \\ 3 Asclepios project-team, Inria Sophia Antipolis, Sophia Antipolis, France
}

\begin{abstract}
Quantitative and qualitative analysis of white matter fibers resulting from tractography algorithms is made difficult by their huge number. To this end, we propose an approximation scheme which gives as result a more concise but at the same time exhaustive representation of a fiber bundle. It is based on a novel computational model for fibers, called weighted currents, characterised by a metric that considers both the pathway and the anatomical locations of the endpoints of the fibers. Similarity has therefore a twofold connotation: geometrical and related to the connectivity. The core idea is to use this metric for approximating a fiber bundle with a set of weighted prototypes, chosen among the fibers, which represent ensembles of similar fibers. The weights are related to the number of fibers represented by the prototypes. The algorithm is divided into two steps. First, the main modes of the fiber bundle are detected using a modularity based clustering algorithm. Second, a prototype fiber selection process is carried on in each cluster separately. This permits to explain the main patterns of the fiber bundle in a fast and accurate way.
\end{abstract}

\section{Introduction}

Tractography from diffusion-weighted magnetic resonance imaging (DW-MRI) is a technique capable to virtually map the neural architecture of the human brain white matter (WM). This method is very useful for a better characterization of neurological diseases, for surgical planning or for the study of anatomofunctional relationships, for example. Tractography methods result in a set of 3D tracts that are commonly referred to as "fibers" which represent an estimate of the trajectory of large groups of neural fibers. WM fibers are traced starting from points, called seeds, inside one or more voxels and they are constituted by segments connecting different voxels. Tractography algorithms can be divided into two classes: deterministic and probabilistic. Deterministic methods reconstruct the fiber following the principal direction given by the diffusion model inside each voxel while the probabilistic ones use a randomly perturbed version of the main fiber direction. The idea behind probabilistic methods is to start many 
fibers from every seed in order to obtain maps of connectivity that are related to the probability that a certain voxel is connected to the starting seed. The choice of the starting voxels depends on the application and it ranges from the whole-brain to particular areas of the gray matter (ROI). One can also choose a second ROI as ending area in order to retrieve only the fibers connecting two precise areas of the gray matter. This paper focuses on such sets of fibers, called fiber bundles, which connect the cortical surface to the basal ganglia. A fiber is therefore characterised by its pathway between voxels and by the anatomical locations of its starting and ending point. Tractography algorithms usually result in a considerable amount of fibers. This makes difficult the development of efficient computational methods and the visualisation and interpretation of the brain connections that have been captured. To this end, we propose a novel approximation scheme for fiber bundles which reduces the number of tracts to analyse conserving almost all the information related to the fibers pathway (geometry) and to the distribution of the fibers endpoints (connectivity).

The core idea is to approximate a fiber bundle $B$ with a set of weighted prototypes $\left\{\tau_{i} M_{i}\right\}$, chosen among the fibers, which represent ensembles of similar fibers. The weights $\left\{\tau_{i}\right\}$ are linked to the number of fibers approximated by the prototypes $\left\{M_{i}\right\}$. In order to do that, we need an appropriate dissimilarity measure based on both the geometry and the connectivity of a fiber. Usual dissimilarity measures present in the literature consider only the geometry of the fibers, like the Hausdorff distance [5], the Chamfer distance (or modified versions of it) 56812 , the total square loss between two fibers represented as Gaussian mixture model [13] or Fourier descriptors [10]. Other dissimilarity measures consider only the ending points of the fibers [11] or are based on the voxel space [12 14]. Here we propose a novel dissimilarity measure which considers both the pathway and the anatomical locations of the termini of the fibers. It can be seen as an extension of the framework of currents [3] and we have called it: weighted currents. As usual currents, it does not need point-to-point correspondence between fibers, it has a closed form and easy to compute expression and it can be used to compare fiber bundles. In addition, two fibers modelled as weighted currents are considered similar if and only if their pathways are alike and their endpoints are close to each other. Fibers are considered as vectors in the space of weighted currents. This permits to see a fiber bundle $B$ as the sum of all its fibers $F_{j}: B=\sum_{j} F_{j}$ and its approximation as a weighted sum: $\sum_{i=1}^{K} \tau_{i} M_{i}$.

The proposed approximation scheme is based on the minimization of the residual error between $B$ and $\left\{\tau_{i} M_{i}\right\}$ which can be easily written as: $\| B-$ $\sum_{i=1}^{K} \tau_{i} M_{i} \|_{W_{*}}^{2}$ in the framework of weighted currents. The final goal is to find the smallest set of prototypes which minimizes this approximation error. An exhaustive analysis of all the possible combinations of prototypes is not feasible and therefore we propose a greedy algorithm. Our strategy is first to decompose a fiber bundle into modes, and then approximate every mode assuming that its fibers follow a gaussian distribution. In order to do that, the fiber bundle is first divided into independent clusters, which can be seen as different modes, using a clustering algorithm. Afterwards, the prototype fiber selection process is carried 
on in each cluster separately. This permits a fast (parallel computing), concise and at the same time exhaustive approximation of the fiber bundle.

Usual clustering approaches for WM tracts are based on hierarchical [8|5 12 13], spectral 611] or EM [7] techniques. In many cases one needs to fix in advance parameters like the number or the size of the clusters. A solution, which does not require parameter setting, is to employ one of the quality functions in the field of networks community detection: the modularity 9]. It determines how good a cluster division is by comparing the similarity of fibers inside clusters with respect to the similarity of fibers between clusters. Optimization methods based on the maximization of this quality function permit to automatically find the number of clusters and how to divide the fiber bundle into clusters.

Eventually, the prototypes are chosen by selecting the fibers that minimize the residual error between $B$ and $\left\{\tau_{i} M_{i}\right\}$ in the spirit of PCA (Principal Component Analysis) exploiting the fact that every mode is assumed to be gaussian (unimodal). This permits to explain and approximate almost the entire variability of the fiber bundle in a fast and accurate way.

\section{Approximation Scheme for WM Fiber Bundles}

In the first paragraph we introduce the concept of weighted currents from a formal mathematical point of view. Afterwards, we present the two steps of the approximation scheme based on weighted currents: the modularity optimization clustering algorithm and the prototype fiber selection.

Weighted Currents can be seen as an adaptation of the functional currents presented in [4] where the "functional signal" attached to each fiber is the anatomical location of its starting and ending point. Let $X$ be a WM tract (an oriented and rectifiable curve in $\boldsymbol{R}^{3}$ ) which can be modelled as a polygonal line of $N$ segments and $f^{c}$ and $f^{b}$ two 3 D vectors in $M=\boldsymbol{R}^{3} \times \boldsymbol{R}^{3}$ containing the coordinates of the two extremities. For example, $f^{c}$ might represent the extremity of the fiber on the cortical surface and $f^{b}$ on the basal ganglia. In practice, $f^{c}$ and $f^{b}$ could be the mean values of the last $n$ points, i.e. $n=3$, if the extremities are supposed to be in an area with a low SNR. The tract X, exactly as for currents, can be seen as a discrete weighted current via: $C_{X}(w)=\sum_{i=1}^{N} w\left(x_{i}, f^{c}, f^{b}\right)^{T} \alpha_{i}$ where $x_{i}$ and $\alpha_{i}$ are the centres and the tangent vectors of the $N$ segments respectively and $w$ is a vector field belonging to a reproducing kernel Hilbert space (RKHS) $W$ on $\boldsymbol{R}^{3} \times M$. A natural way to build the kernel associated to $W$ is as the tensor product of kernels defined separately in the geometrical space $\boldsymbol{R}^{3}$ and in the "functional" space $M$. This means that the inner product in the framework of weighted currents between two tracts $X\left(C_{X}(w)=\sum_{i=1}^{N} w\left(x_{i}, f^{c}, f^{b}\right)^{T} \alpha_{i}\right)$ and $Y\left(C_{Y}(w)=\sum_{j=1}^{M} w\left(y_{j}, t^{c}, t^{b}\right)^{T} \beta_{j}\right)$ can be defined as:

$$
\left\langle C_{X}, C_{Y}\right\rangle_{W *}=K_{c}\left(f^{c}, t^{c}\right) K_{b}\left(f^{b}, t^{b}\right) \sum_{i=1}^{N} \sum_{j=1}^{M} \alpha_{i}^{T} K_{g}\left(x_{i}, y_{j}\right) \beta_{j}
$$

where $K_{c}, K_{b}$ and $K_{g}$ are Gaussian kernels parametrized by their scale parameters: $\lambda_{c}, \lambda_{b}$ and $\lambda_{g}$ respectively. The underlined part is the inner product 
between usual currents which measures overall differences in the pathway of the two fibers. $\lambda_{g}$ defines the range of interactions between the points of $X$ and $Y$. The multiplication by the new terms $K_{c}$ and $K_{b}$ means that two fibers are considered similar if the pathways are similar, like in usual currents, but also if the endpoints of the two fibers are at a distance smaller than $\lambda_{c}$ on the cortical surface and than $\lambda_{b}$ on the basal ganglia (see Fig 11). The space of weighted currents is a vector space which implies that a fiber bundle $B$ is seen as the sum of its fibers $F_{i}: C_{B}=\sum_{i}^{N} C_{F_{i}}$ and that it is also possible to compute the mean of a fiber bundle: $C_{\bar{F}}=\frac{1}{N} \sum_{i}^{N} C_{F_{i}}$. In the following, we will assume that each fiber is modelled as weighted current writing simply $F$ instead than $C_{F}$. As we will see, our processing will be calculated using only simple computations of the Gram matrix $\Gamma=\left\{\left\langle F_{i}, F_{j}\right\rangle_{W *}\right\}_{i, j=1, \ldots, N}$.
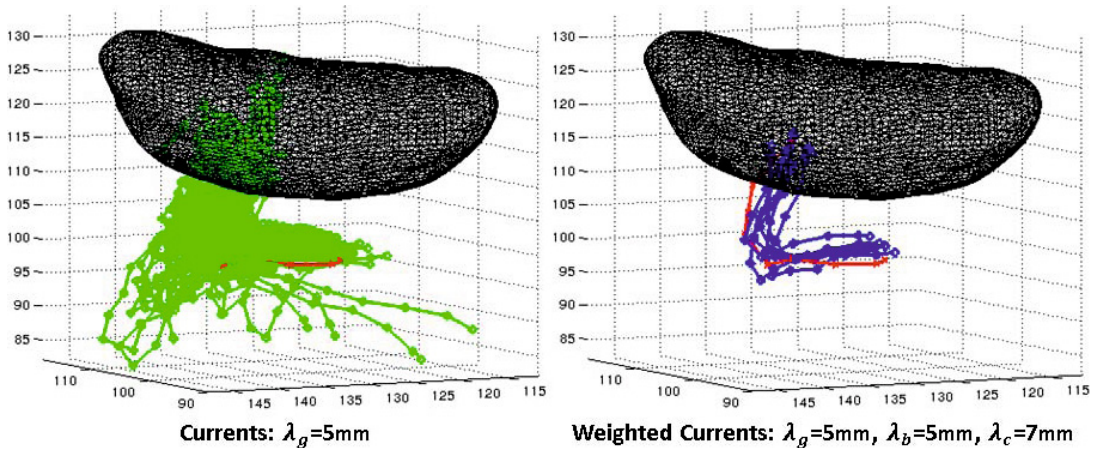

Fig. 1. Tracts that have an angle smaller than 45 degrees with the red one using currents (green, \#118) and weighted currents (blue, \#8). Fibers belong to a thalamocortico bundle of the right hemisphere resulting from a probabilistic tractography (see Sec 3). Green tracts are more than the blue ones and they are also more spread, connecting anatomical locations far from the ones of the red fiber. This shows why weighted currents are more suitable for clustering and approximating fiber bundles.

Modularity Optimization Clustering. A fiber bundle is seen as a sum of vectors in the space of weighted currents, each vector representing a fiber. Every vector can then be considered as a vertex of a weighted graph where the weighted edges are the inner products between every couple of fibers. The first step of the proposed algorithm consists in finding clusters in this set of vectors representing the main modes of the fibers distribution. We use a clustering algorithm based on the maximization of a quality function $Q$ called Modularity [9]:

$$
Q=\sum_{c=1}^{N_{C}}\left\{\left\|\sum_{i \in c} F_{i}\right\|_{W^{*}}^{2}\left\|\sum_{j \notin c} F_{j}\right\|_{W^{*}}^{2}-\left(\sum_{i \in c} \sum_{j \notin c}\left\langle F_{i}, F_{j}\right\rangle_{W^{*}}\right)^{2}\right\}
$$

where $N_{C}$ is the number of clusters. This equation can be easily rewritten in terms of the Gram matrix $\Gamma$ as: $\mathrm{Q}=\sum_{c=1}^{N_{C}}\left(s_{c}^{T} \Gamma s_{c}\right)\left(\left(1-s_{c}\right)^{T} \Gamma\left(1-s_{c}\right)\right)-\left(s_{c}^{T} \Gamma\left(1-s_{c}\right)\right)^{2}$ where 
$s_{c}(k)=1$ if $F_{k}$ belongs to cluster $c$ and 0 otherwise. In the simple case of $N_{C}=2$ this equation can be rewritten as: $Q=\left\|\bar{F}_{1}\right\|_{W^{*}}^{2}\left\|\bar{F}_{2}\right\|_{W^{*}}^{2}-\left\langle\bar{F}_{1}, \bar{F}_{2}\right\rangle_{W^{*}}^{2}$ where $\bar{F}_{1}$ and $\bar{F}_{2}$ are the means of the two clusters. Maximizing Q means therefore looking for two clusters whose means are as orthogonal as possible and at the same time their norms should be as close as possible. This can be generalized to $N_{C}$ clusters by saying that the goal of modularity is to create clusters with balanced norms characterised by fibers orthogonal to the fibers in the other clusters and parallel to the fibers in their own cluster. Unfortunately, exact modularity optimization is a NP-complete problem. The "Louvain" algorithm [9] is a greedy solution divided into two steps which are repeated iteratively. At the beginning every WM tract forms a different cluster. The first part consists of associating every WM tract to all its neighbour clusters finding the one that leads to the largest increase in $Q$. This step is repeated until no change would produce an increase in $Q$. The second part consists in merging all the WM tracts of one cluster in one single supervertex. Two supervertices have a weighted edge equal to the sum of all the inner products between the fibers of the initial clusters. The two steps are repeated until no change would produce an increase in $Q$. At the end of this process the fiber bundle is separated into different clusters without fixing in advance neither the number of clusters nor their size.

Prototype Fiber Selection. The goal of the Prototype Fiber Selection (PFS) process is to concisely represent the fiber bundle $B$ with a set of weighted prototypes $\left\{\tau_{i} M_{i}\right\}$ chosen among the fibers. If we wanted only one weighted prototype $\tau_{1} M_{1}$ which minimizes $\left\|B-\tau_{1} M_{1}\right\|_{W_{*}}^{2}$, it would be: $M_{1}=\operatorname{argmax}_{F_{z}}\left\langle B, \frac{F_{z}}{\left\|F_{z}\right\|}\right\rangle_{W_{*}}^{2}$ $=\operatorname{argmax}_{F_{z}} N^{2}\left\langle\bar{F}, \frac{F_{z}}{\| F_{z}}\right\rangle_{W_{*}}^{2}$ with $\tau_{1}=\frac{\left\langle B, M_{1}\right\rangle}{\left\|M_{1}\right\|^{2}}$. This means that we would look for the fiber most similar to the average of the bundle. This scheme works fine only in a uni-modal setting (i.e. gaussian) but not in a multi-modal one since the fiber chosen would be the one closest to the center of the different modes. If the modes are far from each other the fiber chosen could be also considered as an outlier. This is why it is fundamental finding the main modes of the bundle through the previous clustering step. Once defined the main modes, a PFS is performed independently on each one of them. One prototype is not sufficient to explain the whole cluster. So, as for instance in PCA, we remove from each fiber $\left(F_{i}\right)$ its orthogonal projection onto the prototype $\left(\pi\left(F_{i}\right)=\frac{\left\langle F_{i}, M_{1}\right\rangle M_{1}}{\left\|M_{1}\right\|^{2}}\right)$ and we select, in this new representation $\left(r\left(F_{i}\right)=F_{i}-\pi(F i)\right)$, the fiber most similar to the new average as second prototype $\left(M_{2}=\operatorname{argmax}_{r\left(F_{z}\right)} N^{2}\left\langle r(\bar{F}), \frac{r\left(F_{z}\right)}{\left\|r\left(F_{z}\right)\right\|}\right\rangle^{2}\right)$. We iterate this process for each cluster $C_{j}$ until: $\left\|C_{j}-\sum_{i=1}^{K_{j}} \tau_{i} M_{i}\right\|_{W_{*}} \leq \gamma\left\|C_{j}\right\|_{W_{*}}$. At iteration $k$, the $k$ weights $\left\{\tau_{i}\right\}$ are computed by the orthogonal projection of the cluster $C_{j}$ to the space spanned by the selected $k$ prototypes $\left\{M_{i}\right\}$. It is important to notice that all these computations are based on the Gram matrix $\Gamma$ of the fiber bundle, also when computing a new prototype: $\left\langle r\left(F_{i}\right), r\left(F_{j}\right)\right\rangle=$ $\left\langle F_{i}, F_{j}\right\rangle-\frac{\left\langle F_{i}, M\right\rangle\left\langle F_{j}, M\right\rangle}{\|M\|^{2}}=\Gamma_{i j}-\frac{\Gamma_{i M} \Gamma_{j M}}{\left\|\Gamma_{M M}\right\|^{2}}$. After selecting the prototypes in each cluster, the weights are recomputed by the orthogonal projection of the whole bundle $B$ to the entire set of prototypes in order to retrieve the correct values also for the prototypes close to the boundary between two different clusters. 


\section{Experiments and Discussion}

We illustrate the algorithm on 36 fiber bundles from 6 subjects connecting the cortex to thalamus (12), putamen (12) and caudate (12) of the right hemisphere using both deterministic and probabilistic tractography estimated from HARDI data (1] and references therein). The fiber bundles include the commisural fibers which have been truncated at the interhemispheric fissure [1. The segmentation of the sub-cortical structures and of the cortex is done using FSL and FreeSurfer respectively [2]. The result of the clustering and the prototype representation for both deterministic and probabilistic tractography on a cortico-thalamus fiber bundle are shown in Fig 2 Other results are presented in the supplementary material. In the deterministic case only 491 fibers out of 9525 have been chosen as prototypes ( $\sim 5 \%)$ in order to explain $90 \%$ of $\|B\|_{W_{*}}$ while with the probabilistic tractography only 2652 fibers out of 10410 have been used as prototypes $(\sim 25 \%)$ to explain $85 \%$ of $\|B\|_{W_{*}}$. The average reduction in the deterministic case is $4.7 \%$ explaining $90 \%$ of $\|B\|_{W_{*}}$ and $24.6 \%$ in the probabilistic case explaining $84 \%$ of $\|B\|_{W_{*}}$. Once calculated the Gram matrix ( $\left.120 \mathrm{~min}\right)$, the computation of the whole approximation scheme is very fast: about $40 \mathrm{sec}$ for the clustering and $30 \mathrm{sec}$ for the PFS in a PC, Intel Xeon, 4 cores, $3.20 \mathrm{GHz}$ using 10410 fibers. Fig 3 shows the same bundle of Fig 2 but its goal is to point out the differences between our approximation scheme and a uniform downsampling resulting in the same number of tracts. The probability densities of the endpoints of the original fibers on both cortex and thalamus are more similar to the ones of our scheme than with a uniform downsampling. In fact, some small modes are lost with the uniform downsampling. Probability densities have been computed using gaussian kernels, taking into account the weights $\tau_{i}$ of the prototypes
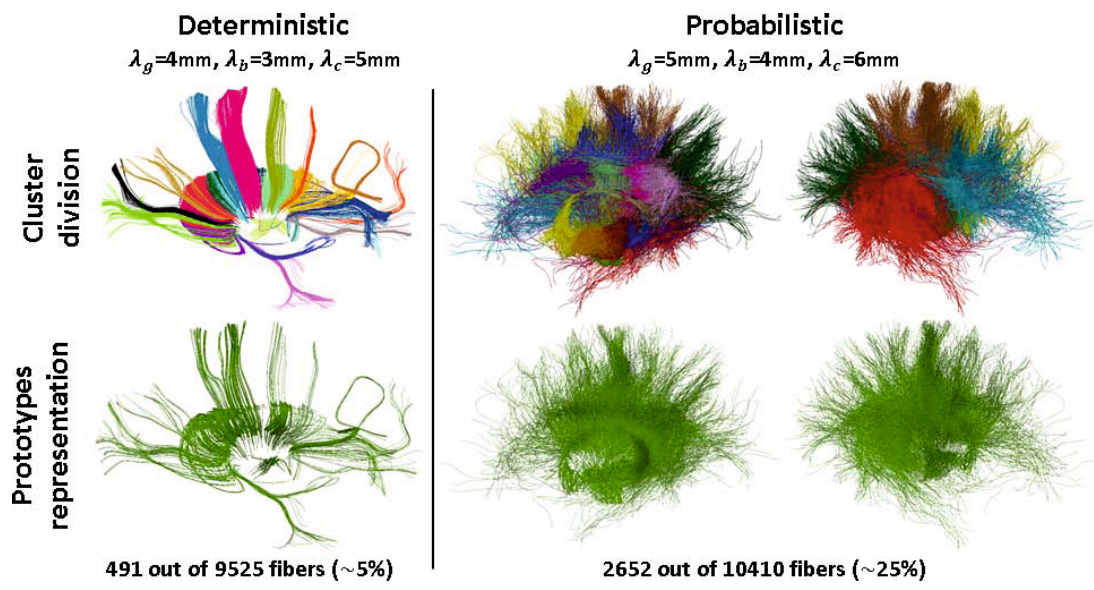

Fig. 2. First row: clusters of the right thalamo-cortico bundle highlighted in different colors. Second row: resulting prototypes visualized as tubes whose radii are proportional to their weights $\tau_{i}$. The two columns on the right show the same fiber bundle from both lateral views. 

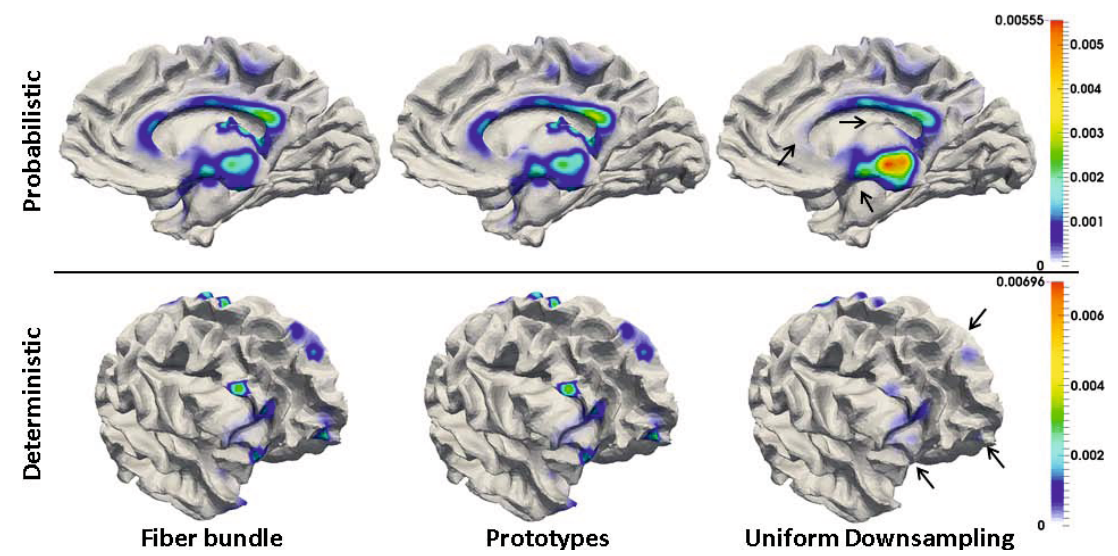

Fig. 3. Probability densities of the endpoints of the fibers using the right thalamocortico fiber bundle, prototypes $P$ and an equal number of fibers coming from a uniform downsampling $U$ of the original fiber bundle. $U$ is a poorer approximation of the original density than $P$ since it reveals fewer modes, as shown by the arrows.

for our approximation. The Kolmogorov-Smirnov test fails to show statistically significant differences between the densities of the original fibers and of the weighted prototypes and in most of the cases it finds significant differences at the $5 \%$ level between densities from original fibers and uniform downsampling.

\section{Conclusions}

We have presented here a new approximation scheme for white matter fiber bundles which results in a concise representation maintaining almost all the information related to the pathways of the fibers and to the locations of the fiber endpoints. It is based on a new computational model for fiber bundles, called weighted currents, which permits to compare fibers considering both their pathways and the locations of their endpoints. Moreover, it allows to treat fibers as vectors. We have tested this method on fiber bundles resulting from both deterministic and probabilistic tractography showing that the number of tracts to analyse can be reduced up to $3 \%$ of the initial number of fibers in the deterministic case and up to $17 \%$ in the probabilistic case, explaining in both cases more than $84 \%$ of the norm of the fiber bundles. We have also shown that the connectivity information, seen as the probability density of the fibers endpoints onto the gray matter, is more preserved with the proposed method than using a uniform downsampling. This information is important in many neuro-anatomical studies since it could be used, for instance, to characterise neurodevelopmental disorders [2]. Future works will include this new representation into registration and atlas construction methods in order to find changes in white matter organization across subjects. Another possible development could be to use this method for the white matter segmentation problem [612]. 
Acknowledgements. The research leading to these results has received funding from the program "Investissements d'avenir" ANR-10-IAIHU-06.

\section{References}

1. Marrakchi-Kacem, L., Delmaire, C., Guevara, P., Poupon, F., Lecomte, S., Tucholka, A., Roca, P., Yelnik, J., Durr, A., Mangin, J., Lehéricy, S., Poupon, C.: Mapping Cortico-Striatal Connectivity onto the Cortical Surface: A New TractographyBased Approach to Study Huntington Disease. PLoS One 8, e53135 (2013)

2. Worbe, Y., Gerardin, E., Hartmann, A., Valabrègue, R., Chupin, M., Tremblay, L., Vidailhet, M., Colliot, O., Lehéricy, S.: Distinct structural changes underpin clinical phenotypes in patients with Gilles de la Tourette syndrome. Brain 133, 3649-3660 (2010)

3. Durrleman, S., Fillard, P., Pennec, X., Trouvé, A., Ayache, N.: Registration, Atlas Estimation and Variability Analysis of White Matter Fiber Bundles Modeled as Currents. NeuroImage 55, 1073-1090 (2011)

4. Charon, N., Trouvé, A.: Functional Currents: A New Mathematical Tool to Model and Analyse Functional Shapes. J. Math. Imaging Vis. 48, 413-431 (2014)

5. Gerig, G., Gouttard, S., Corouge, I.: Analysis of Brain White Matter via Fiber Tract Modeling. In: 26th IEEE EMBS, vol. 2, pp. 4421-4424. IEEE Press, New York (2004)

6. O'Donnel, L.J., Westin, C.F.: Automatic Tractography Segmentation Using a HighDimensional White Matter Atlas. IEEE Trans. Med. Imaging 26, 1562-1575 (2007)

7. Maddah, M., Grimson, W., Warfield, S., Wells, W.: A unified framework for clustering and quantitative analysis of white matter fiber tracts. Med. Image Anal. 12, 191-202 (2008)

8. Zhang, S., Correia, S., Laidlaw, D.: Identifying White-Matter Fiber Bundles in DTI Data Using an Automated Proximity-Based Fiber Clustering Method. IEEE Trans. Vis. Comput. Graph. 14, 1044-1053 (2008)

9. Blondel, V.D., Guillaume, J.L., Lambiotte, R., Lefebvre, E.: Fast unfolding of communities in large networks. J. Stat. Mech. Theory Exp. 10, P10008 (2008)

10. Batchelor, P.G., Calamante, F., Tournier, J., Atkinson, D., Hill, D.L., Connelly, A.: Quantification of the shape of fiber tracts. Magn. Reson. Med. 55, 894-903 (2006)

11. Brun, A., Park, H.J., Knutsson, H., Westin, C.F.: Coloring of DT-MRI Fiber Traces Using Laplacian Eigenmaps. In: Moreno-Díaz Jr., R., Pichler, F. (eds.) EUROCAST 2003. LNCS, vol. 2809, pp. 518-529. Springer, Heidelberg (2003)

12. Guevara, P., Poupon, C., Rivire, D., Cointepas, Y., Descoteaux, M., Thirion, B., Mangin, J.: Robust clustering of massive tractography datasets. NeuroImage 54, 1975-1993 (2011)

13. Liu, M., Vemuri, B.C., Deriche, R.: Unsupervised Automatic White Matter Fiber Clustering Using a Gaussian Mixture Model. In: 9th IEEE ISBI, pp. 522-525. IEEE Press, New York (2012)

14. Tunç, B., Smith, A.R., Wasserman, D., Pennec, X., Wells, W.M., Verma, R., Pohl, K.M.: Multinomial probabilistic fiber representation for connectivity driven clustering. In: Gee, J.C., Joshi, S., Pohl, K.M., Wells, W.M., Zöllei, L. (eds.) IPMI 2013. LNCS, vol. 7917, pp. 730-741. Springer, Heidelberg (2013) 